\title{
Material Characterization of Single Crystalline Cu Subjected to High Strain Rates and High Temperatures for Multiscale Simulation
}

\author{
Yujin Seong', Youngkyu Kim, Im Doo Jung', Sungho Kim², See Jo Kim³, Seong-Gon Kim, \\ Hak Jun Kim ${ }^{5}$, and Seong Jin Park,* \\ ${ }^{1}$ Department of Mechanical Engineering, Pohang University of Science and Technology, Pohang 37673 , \\ Republic of Korea \\ ${ }^{2}$ Center for Computational Sciences, Mississippi State University, MS 39762, USA \\ ${ }^{3}$ Department of Mechanical Design, Andong National University, Andong 36729, Republic of Korea \\ ${ }^{4}$ Department of Physics and Astronomy, Mississippi State University, MS 39762, USA \\ ${ }^{5}$ Agency for Defense Development, Daejeon 34186, Republic of Korea
}

\begin{abstract}
The material characterization of single crystalline $\mathrm{Cu}$ columns was numerically carried out at the submicroscopic level. A molecular dynamics (MD) simulation was employed using the embedded-atom method (EAM) interatomic potential between a pair of $\mathrm{Cu}$ atoms to describe the interactions among $\mathrm{Cu}$ atoms. First, the relationship between mechanical properties and factors affecting their behavior were numerically investigated using a crystal structure including several defects. The factors were specimen size, strain rate, and temperature. As the specimen size increased the normalized yield stress decreased, which was similar to results obtained at other length-scale. The yield stress tended to lead to exponential strain rate-hardening and a linear temperature-softening. Next, material characterization was conducted based on these results. These computational results can lead to the development of an in silico platform to characterize material properties and MD simulation can lay the groundwork for multi-scale modeling and simulation.
\end{abstract}

(Received June 16, 2017; Accepted June 29, 2017)

Keywords: molecular dynamics simulation, embedded-atom method, copper, material characterization, multiscale simulation

\section{INTRODUCTION}

Nano-scale research has expanded enormously, resulting in promising applications based on the enhanced physical properties found at the nanoscale [1-3]. Among this research, numerous studies have been conducted to understand the mechanical behaviors of metal nano-wires [4-8]. Such metallic materials have many applications, including as nanoscale tips for scanning tunneling microscopes (STM) and atomic force microscopes (AFM) [8], and nanoscale wiring in integrated circuits [9].

Furthermore, nanoscale studies are considered as a useful way of approaching multiscale simulation to identify material properties across various ranges of length and time scales [4,10-12]. Silva et al. [12] discussed diverse problems to

*Corresponding Author: Seong Jin Park

[Tel: +82-54-279-2182, E-mail: sjpark87@postech.ac.kr]

Copyright (c) The Korean Institute of Metals and Materials examine the potential of multiscale modelling. Abraham et al. [13] used a hybrid simulation method using quantummechanical derivations with molecular dynamics simulations, in which they used hand-shaking algorithms between the finite-element and MD regions, and between MD and the quantum tight-binding regions.

In this paper, we have studied the mechanical properties of nanoscale copper column and have carried out material characterization at the atomistic scale to further investigate the multiscale simulation of alloys containing copper atoms.

\section{MODELING \& SIMULATIONS}

Molecular dynamics (MD) simulations were carried out using LAMMPS [14,15], which is one of the open sources programs for MD simulations. The embedded-atom method (EAM) approach was employed to describe the motion of 


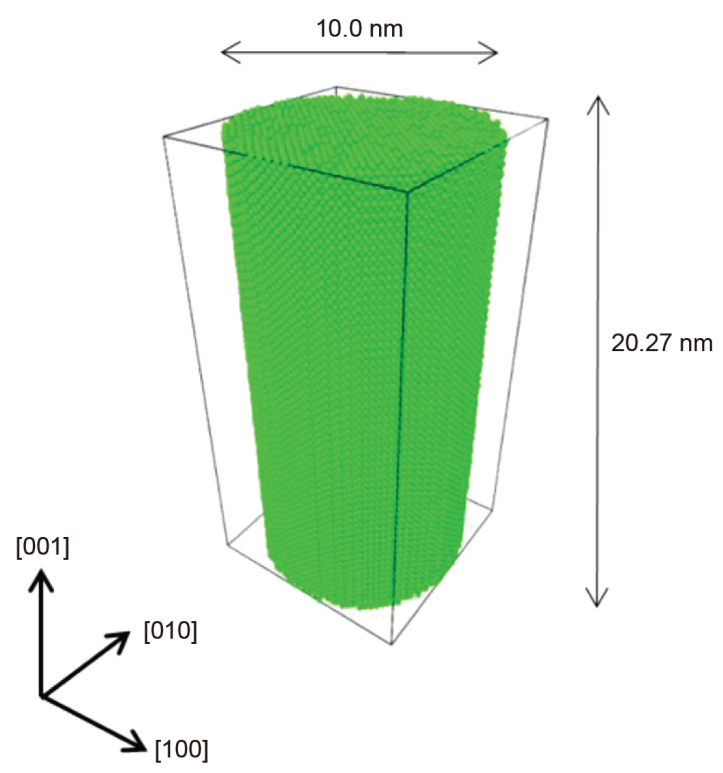

Fig. 1. A specimen for tensile test.

atoms. In the EAM approach [16,17], the total energy of a system of atoms is written as the sum of the atomic energy,

$$
E=\sum_{i} E_{i}
$$

with the atomic energy being the sum of two contributions,

$$
E_{i}=\frac{1}{2} \sum_{j \neq i} V\left(r_{i j}\right)+F\left(\rho_{i}\right)
$$

where $V\left(r_{i j}\right)$ the an interatomic pairwise potential, and $F\left(\rho_{i}\right)$ is the embedding energy function of the local "atomic density" at the site of atom $i$. The interatomic pairwise potential represents the interaction between an individual atom and its neighbor as a function of the relative distance $r_{i j}$. The embedding energy describes the interaction among the electronic orbitals of the atoms. A non-dimensional term $\rho_{i}$ is also considered to be the contribution of each neighbor atom to the electronic density of a particular atom,

$$
\rho_{i}=\sum_{j \neq i} \rho\left(r_{i j}\right),
$$

In this paper, the EAM parameters of $\mathrm{Cu}$ proposed by
Mishin et al. [18] were used for the simulations.

During the tensile test simulation, the atomic stress on the $\alpha$-plane and in the $\beta$-direction was calculated using the Virial theorem [19], which takes the form

$$
\sigma_{\alpha \beta}=\frac{1}{N} \sum_{i}\left[-\frac{m_{i} v_{i}{ }^{\alpha} v_{i}{ }^{\beta}}{\Omega_{i}}+\frac{1}{2 \Omega} \sum_{j \neq i} \frac{\partial \phi\left(r_{i j}\right)}{\partial r_{i j}} \frac{r_{i j}{ }^{\alpha} r_{i j}{ }^{\beta}}{r_{i j j}}\right. \text { (4) }
$$

where $N$ is the number of atoms, $\Omega_{i}$ the volume of $i$-th atom, $m_{i}$ the mass, $V^{a}$ the velocity component in the $\alpha$ -direction, $r_{i j}$ the relative distance between $i$-th atom and $j$-th atom, respectively. The first term is a kinetic energy component for the $i$-th atom, and the second term is a potential component due to the interatomic force.

\section{RESULTS \& DISCUSSIONS}

A uniaxial tensile test was simulated using the MD simulation with the EAM potential. As shown in Fig. 1, the specimen was a FCC copper nano-column without any defects under tensile loading in the [001] direction. The longitudinal direction of the specimen was set on a periodic boundary condition. The other two orthogonal directions, [100] and [010], had free surfaces. The OVITO program, which is a freely available visualization software developed by Alexander Stukowski at Darmstadt University of Technology in Germany, was used to visualize the simulation results [20].

The characteristic length and normalized yield stress were introduced to evaluate the relationship between the yield stress and factors affecting the material behaviors, such as specimen size, strain rate, and temperature. The characteristic length was defined by the ratio of volume, $V$ and the free surface area, $S: L_{c}=4 S / V$. For example, the $L_{c}$ for a cylindrical specimen is equal to the diameter and the $L_{c}$ for a cubical specimen is equal to the segment length. $L_{c}$ is useful for analyzing the data of various specimen shapes and sizes. The normalized yield stress was defined by the ratio of yield stress to elastic modulus, which is a non-dimensional parameter. 


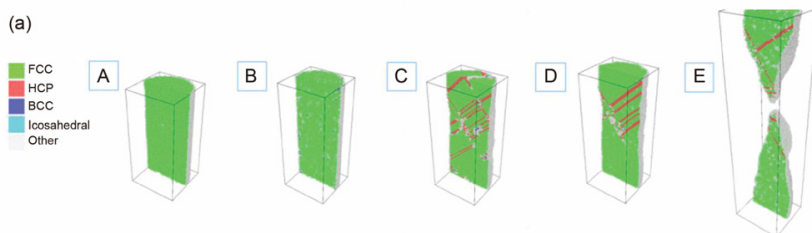

(b)

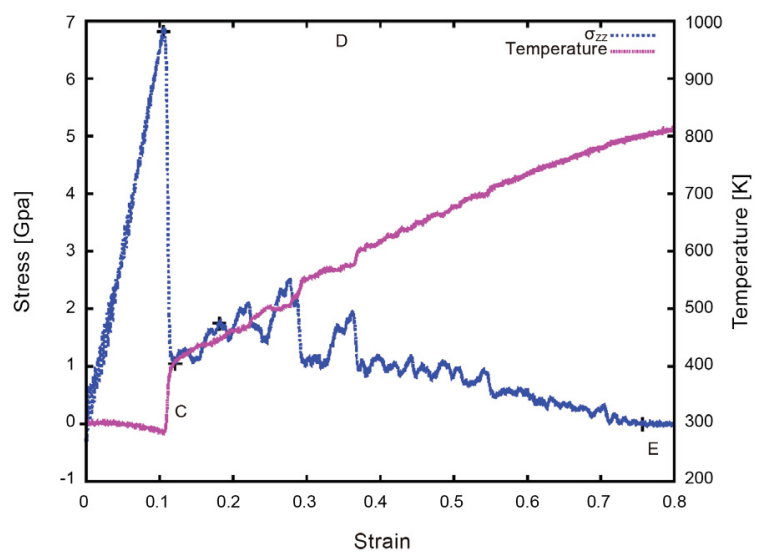

Fig. 2. (a) Evolution of copper nano-column, and (b) stress-strain curve and change of temperature. ' $A$ ' is at initial state, ' $B$ ' at yielding point, ' $C$ ' after first slip, ' $D$ ' at locally highest stress state, and ' $\mathrm{E}$ ' at fracture.

\subsection{Overall tensile behavior}

The uniaxial tension test was simulated with a $10 \mathrm{~nm}$ diameter specimen, a strain rate of $10 \% / S$, and a temperature of $300 \mathrm{~K}$. The yield stress and the elastic modulus were 6.83 $\mathrm{GPa}$ and $65.9 \mathrm{GPa}$, respectively. According to Hertzberg [21], the elastic modulus of a single copper crystal in the [001] direction is equal to $E_{[001]}=66.7 \mathrm{GPa}$. The elastic modulus, which is one of the physical properties of the crystal, was similar to that of the material at macroscale, although the yield stress was different with the one at macroscale.

Figure 2 shows the evolution of the copper nano-column from its initial state to fracture, where necking phenomenon was observed. Figure 2(a) and Figure 2(b) are the crystal structure, and the stress-strain relationship, respectively. After elastic elongation, a sudden slip appeared with a quick drop in stress and a corresponding increase in temperature, due to dislocations in region B-C in Fig. 2. The dislocations were initiated by the smallest Burgers vector in the close-packed directions for the FCC crystal structures, making it easy to rearrange along the plane. Then, recrystallization occurred, which rearranged or eliminated the dislocations in region $\mathrm{C}-\mathrm{D}$
Table 1. Effect of specimen size on mechanical properties.

\begin{tabular}{c|c|c|c}
\hline $\begin{array}{c}\text { Diameter } \\
(\mathrm{nm})\end{array}$ & $\begin{array}{c}\text { Yield stress } \\
(\mathrm{GPa})\end{array}$ & $\begin{array}{c}\text { Young's } \\
\text { modulus } \\
(\mathrm{GPa})\end{array}$ & $\begin{array}{c}\text { Normalized yield } \\
\text { stress } \\
(-)\end{array}$ \\
\hline 5 & 7.00 & 67.0 & 0.1045 \\
\hline 10 & 6.83 & 65.9 & 0.1036 \\
\hline 20 & 6.82 & 66.0 & 0.1033 \\
\hline
\end{tabular}

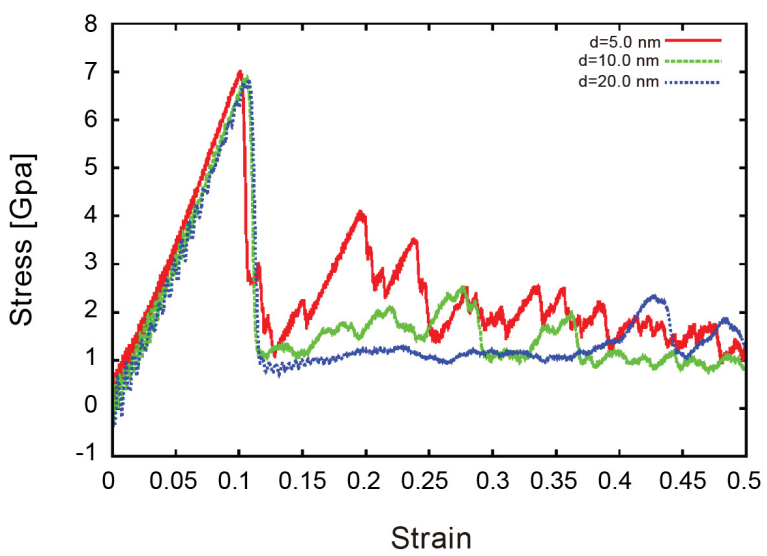

Fig. 3. Effect of specimen size on stress-strain curve at $300 \mathrm{~K}$ with strain rate $1 \mathrm{E} 9 / \mathrm{s}$.

in Fig. 2. This reduced the internal energy of the specimen. The slip-recrystallization cycle repeated until fracture in region D-E in Fig. 2.

\subsection{Size effect}

Next, the effect of specimen size was investigated using the tensile test. The radii of the 3 different specimens were 5 $\mathrm{nm}, 10 \mathrm{~nm}$, and $20 \mathrm{~nm}$ with a ratio of diameter to height of 1:2. Figure 3 and Table 1 show the resulting stress-strain relationships and several properties for a strain rate of $10^{9} / S$ at $300 \mathrm{~K}$, respectively. While the elastic modulus was independent of the specimen size, the yield stress and zigzag curve after the yield point was affected by size. The $5 \mathrm{~nm}$ sample had the highest normalized yield stress among them. As the specimen size increased the normalized yield stress slightly decreased, which was similar to the tendency at other length scales. As the specimen size decreases, the stress-strain curve became more zigzag. Besides, the second peak appeared at a very low strain for the smaller size specimen as compared to the bigger size specimen.

Figure 4 shows representative images of the crystal 
(a)

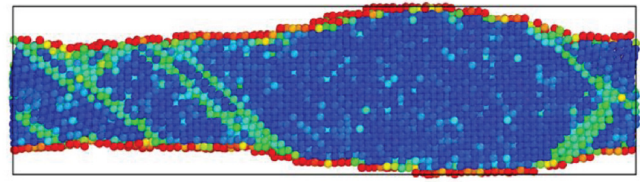

(b)

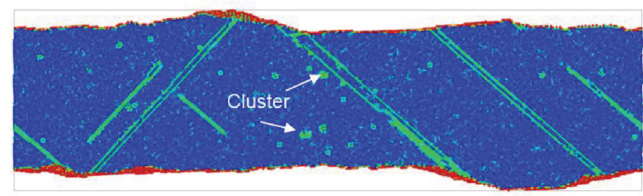

Fig. 4. Crystal structure with (a) diameter $5 \mathrm{~nm}$, and (b) $20 \mathrm{~nm}$.

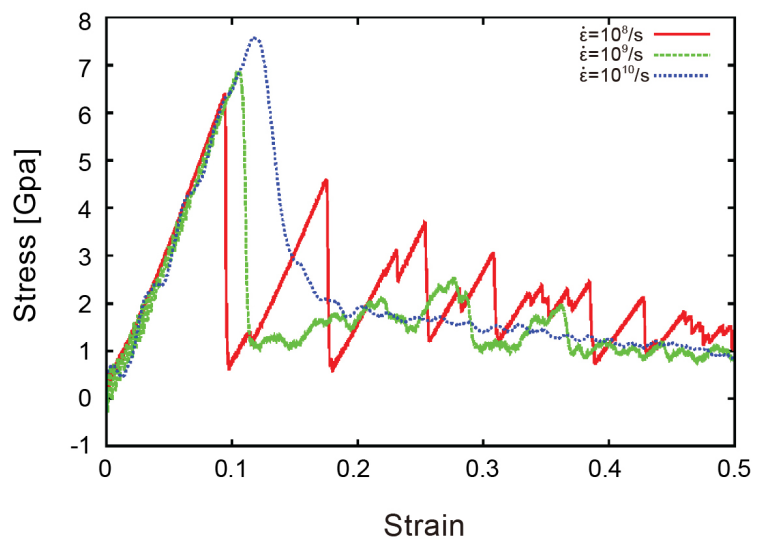

Fig. 5. Effect of strain rate on stress-strain curve of specimen with diameter $10 \mathrm{~nm}$ at $300 \mathrm{~K}$.

structures at different specimen sizes. Clusters can be observed in the cases with $10 \mathrm{~nm}$, and $20 \mathrm{~nm}$ diameter, while the $5 \mathrm{~nm}$ specimen rarely exhibits clusters. Nucleation of the clusters is associated with the ratio of surface to volume. As the specimen size increases, the ratio of surface to volume is reduced. The ratio prevents the nucleation of dislocations, leading to the nucleation of clusters instead of dislocations, to reduce stress in the specimen. The number of clusters increases with increasing specimen size.

\subsection{Strain rate effect}

The strain rate effect was studied for three strain rates $10^{8} /$ $s, 10^{9} / s$, and $10^{10} / s$ at $300 \mathrm{~K}$. As shown in Fig. 5 and Table 2 , the yield stress increases and the zigzag curve is smoother with increased strain rate, while the elastic modulus is independent of the strain rate. The smoothing of the oscillation of the zigzag curve is because there was not
Table 2. Effect of strain rate on mechanical properties.

\begin{tabular}{c|c|c|c}
\hline $\begin{array}{c}\text { Strain rate } \\
(/ \mathrm{s})\end{array}$ & $\begin{array}{c}\text { Yield stress } \\
(\mathrm{GPa})\end{array}$ & $\begin{array}{c}\text { Young's } \\
\text { modulus } \\
(\mathrm{GPa})\end{array}$ & $\begin{array}{c}\text { Normalized yield } \\
\text { stress } \\
(-)\end{array}$ \\
\hline $1 \mathrm{E} 8$ & 6.38 & 65.7 & 0.0971 \\
\hline $1 \mathrm{E} 9$ & 6.83 & 65.9 & 0.1036 \\
\hline $1 \mathrm{E} 10$ & 7.56 & 67.1 & 0.1127 \\
\hline
\end{tabular}

(a)

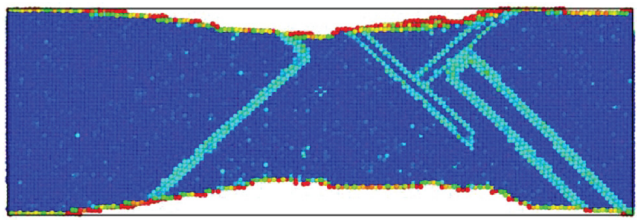

(b)

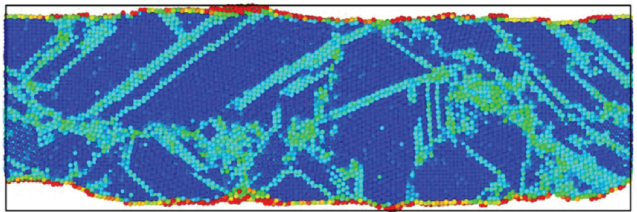

Fig. 6. Crystal structure with (a) strain rate 1E9/s, and (b) 1E10/s.

enough time for the kinetic energy between the deformed atoms to transfer to others at the higher strain rate. Figure 6 shows the crystal structure with two different strain rates. The oscillation mode is associated with the deformation of crystal structure [5]. For $10 \%$, the crystalline structure with the stacking faults and the dislocations was observed to have a clear zigzag curve (Fig. 6(a)). At a higher strain rate, a crystalline structure with amorphous regions was observed, as shown in Fig. 6(b). The higher the strain rate, the weaker the zigzag curve, and the greater the delay in the second pick of the curve, due to more complex defects including amorphization, twinning, and dislocations.

Horstemeyer et al. [4] determined that there are two regions in the yield stress curve for strain rate: the plateau stress region and the stress-increasing regions. In the plateau stress region, the stress is constant and independent of the strain rate. Moreover, the yield stress increases exponentially with respect to strain rate in the macroscale [22]. Taking this into consideration, it was assumed that the yield stress exponentially increases with respect to strain rate at the nanoscale. A critical plateau strain rate was introduced to divide the effect into two regions: the plateau stress region and the exponentially-increasing one. Below the critical 

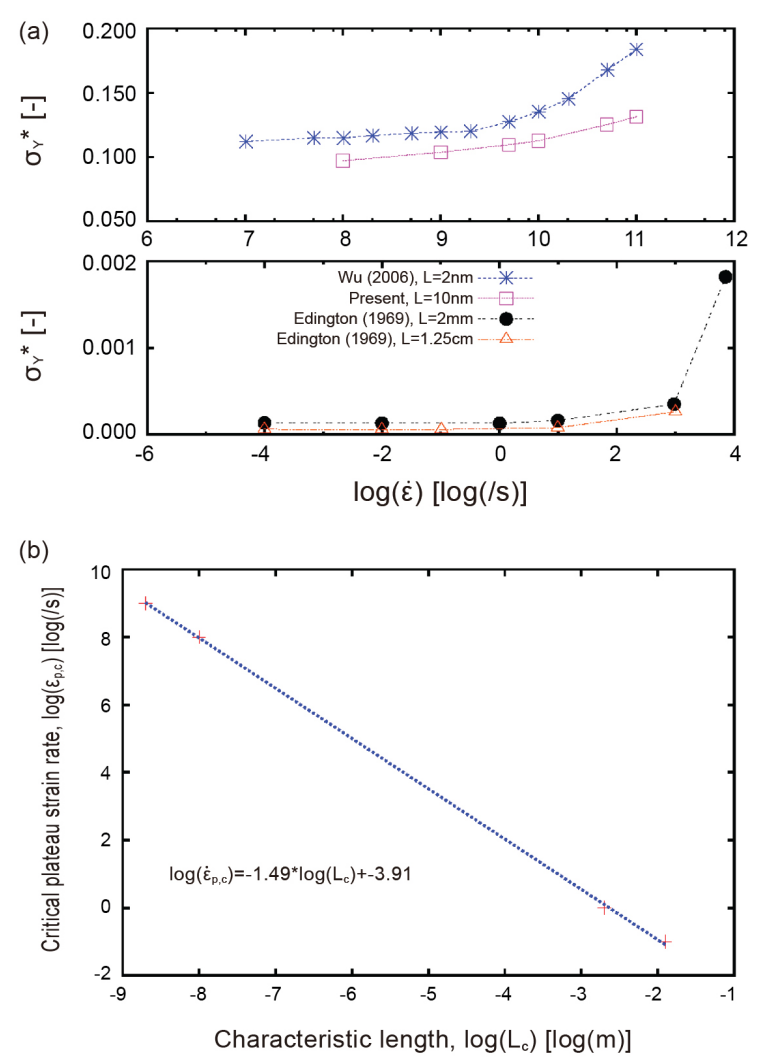

Fig. 7. Critical plateau strain rate with (a) normalized yield stress, and (b) characteristic length.

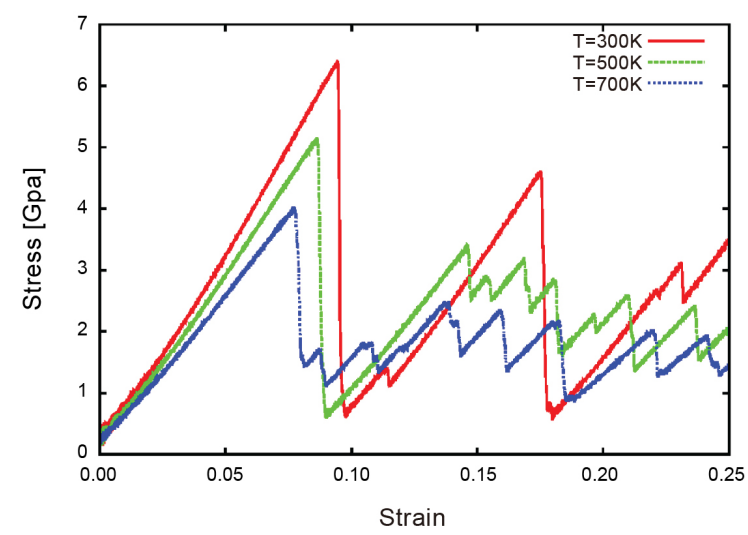

Fig. 8. Effect of temperature on stress-strain curve of specimen with diameter $10 \mathrm{~nm}$ and strain rate $1 \mathrm{E} 8 / \mathrm{s}$.

plateau strain rate, the stress is independent of the strain rate. Above the critical value, the stress increases exponentially with the strain rate. Figure 7(a) is the normalized yield stress with strain rate, where the critical plateau strain rate is dependent on the characteristic length. Numerical/experimental results of the other characteristic length scale $[7,23]$ were
Table 3. Effect of temperature on mechanical properties.

\begin{tabular}{c|c|c|c}
\hline $\begin{array}{c}\text { Temperature } \\
(\mathrm{K})\end{array}$ & $\begin{array}{c}\text { Yield stress } \\
(\mathrm{GPa})\end{array}$ & $\begin{array}{c}\text { Young's } \\
\text { modulus } \\
(\mathrm{GPa})\end{array}$ & $\begin{array}{c}\text { Normalized yield } \\
\text { stress } \\
(-)\end{array}$ \\
\hline 300 & 6.38 & 65.7 & 0.0971 \\
\hline 500 & 5.12 & 57.4 & 0.0892 \\
\hline 700 & 3.98 & 49.3 & 0.0807 \\
\hline
\end{tabular}

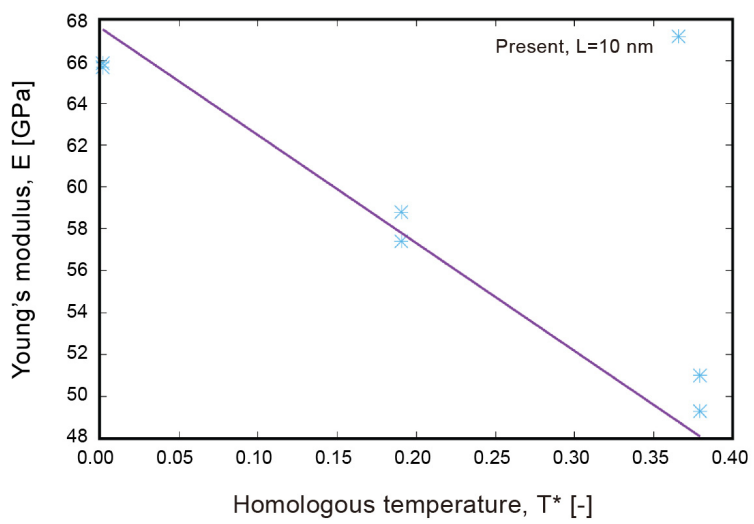

Fig. 9. Young's modulus at various temperatures.

used to determine the relationship between the critical plateau strain rate and the characteristic length, as shown in Fig. 7(b). The relationship between the critical plateau strain rate and the characteristic length is calculated by

$$
\log \left(\varepsilon_{\dot{p}}, c\right)=-1.49 \log L_{c}-3.91
$$

which provides the critical plateau strain rate with the characteristic length, to evaluate the effect of factors independent of the strain rate. The strain rate below the critical value was essentially used to characterize the effects of size and temperature on the material behaviors.

\subsection{Temperature effect}

The nano-column with a diameter of $10 \mathrm{~nm}$ was tested with three temperatures, $300 \mathrm{~K}, 500 \mathrm{~K}$, and $700 \mathrm{~K}$, at a strain rate of $10^{8} / \mathrm{s}$. The higher the temperature, the lower normalized yield stress, and the lower the elastic modulus, as shown in Fig. 8 and Table 3. This is because the atoms have high entropy as the temperature increases and the atoms vibrate with high amplitude. As the atomic motion increases, atomic rearrangement becomes more active. Unlike other factors, the 


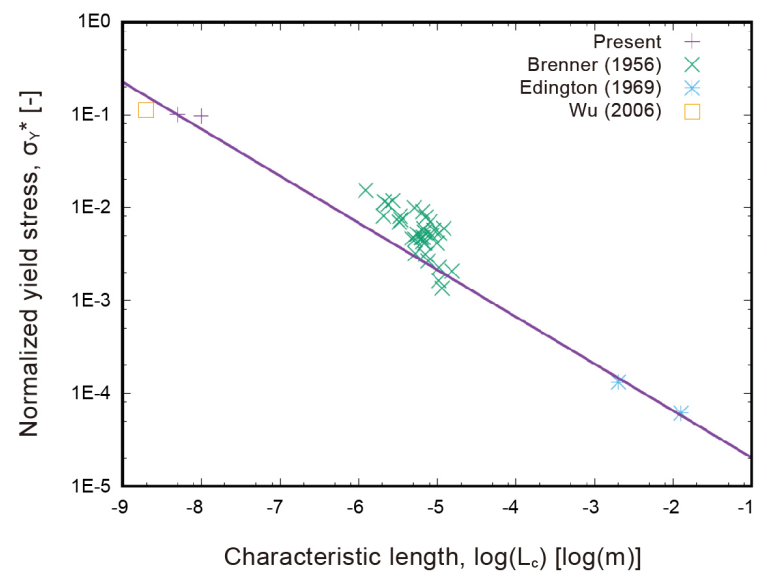

Fig. 10. Relationship between specimen size and yield stress.

elastic modulus linearly decreased with temperature as shown in Fig. 9. The relationship between these parameters can be defined by

$$
E\left(T^{*}\right)=E_{0}\left(1-m T^{*}\right)=67.6\left(1-0.761 T^{*}\right),
$$

where $T^{*}$ is the homologous temperature set to $T^{*}=\left(T-T_{r}\right) /\left(T_{m}-T_{r}\right)$. The subscript ' $\mathrm{r}$ ' and ' $\mathrm{m}$ ' refer to the room temperature and melting temperature, respectively. The unit of elastic modulus is GPa.

\subsection{Material characterization}

The material behaviors of single crystalline copper was characterized using three factors: specimen size, strain rate, and temperature. These effects on the yield stress are discussed in Section 3.2 to 3.4. The yield stress can be expressed as the product of the elastic modulus and the normalized yield stress:

$$
\sigma=E \sigma_{Y}^{*}=E_{0}\left(1-m T^{*}\right)\left(\alpha L_{c}^{\beta}\right)\left(1+C\left(\ln \dot{\varepsilon}^{*}\right)^{p L_{c}^{q}}\right)
$$

Here, $\dot{\epsilon}^{*}$, and $T^{*}$ are non-dimensional parameters. $\dot{\epsilon}^{*}$ is equal to $\dot{\epsilon}^{*}=\dot{\epsilon} / \dot{\epsilon}_{p, c}$, where $\dot{\epsilon}_{p, c}$ is the critical plateau strain rate as shown in Eq. 5. The elastic modulus involves the thermal softening effect. The normalized yield stress is a function of the characteristic length and the strain rate. The unit for $L_{c}$ is a meter. There are seven parameters in the yield
Table 4. Parameters for yield stress

\begin{tabular}{c|c|c|c|c|c|c}
\hline$\alpha$ & $\beta$ & $C$ & $p$ & $q$ & $E_{0}$ & $m$ \\
\hline $6.27 \mathrm{E}-6$ & -0.506 & 0.131 & 2.68 & 0.0193 & $67.6 \mathrm{GPa}$ & 0.761 \\
\hline
\end{tabular}

stress: $E_{0}, m, \alpha, \beta, C, p$, and $q$.

The seven parameters for single crystalline copper were obtained using the experimental and numerical data from the tensile tests, from nanoscale to macroscale. First, the two parameters of the elastic modulus, $E_{0}$ and $m$ were found using Eq. 6. Next, five parameters of the normalized yield stress were obtained by curve-fitting above the critical plateau strain rate. As depicted in Fig. 10, the relationship between the normalized yield stress and the characteristic length has a power law. The micro- and macroscale results have been experimentally obtained by other researchers [23,24]. The parameters are summarized in Table 4. Figures 11 and 12 provide a comparison of the experimental/numerical data and the model used in Eq. 7. The model is well matched with the data. In Fig. 11, the effects of specimen size and strain rate are well expressed in Eq. 7. Figure 12 shows the thermal softening effect, expressed in terms of the elastic modulus only. It is noted that the proposed model can be used for simulating crystal plasticity at the mesoscale, which is one of the steps in multiscale simulation. However, there are limitations in that Eq. 7 is restricted for the single crystalline case only.

\section{CONCLUSION}

The material behavior of a single crystalline $\mathrm{Cu}$ column was characterized by specimen size, strain rate, and temperature. First, the relationship between the mechanical properties and three factors affecting the behavior, specimen size, strain rate, and temperature, were numerically investigated using a crystal structure including several defects. Elastic modulus was found to be only dependent only on temperature, while yield stress was dependent on all three factors. As the specimen size increased the yield stress decreased, which was also found to be the case at other length-scales. Also, zigzag curves after the yield point weakened and clustering in the crystal structure was observed 
(a)

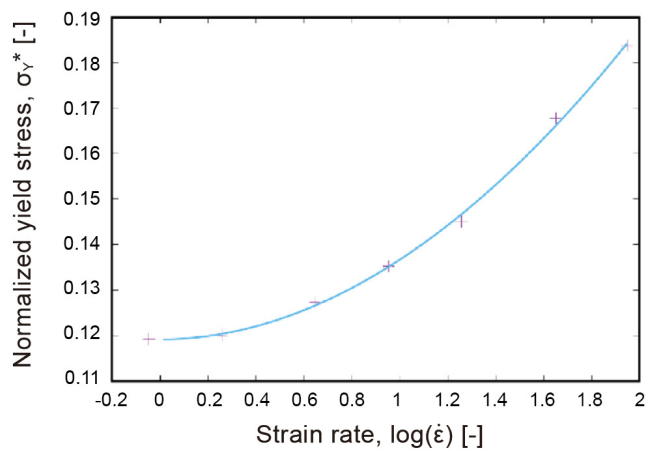

(c)

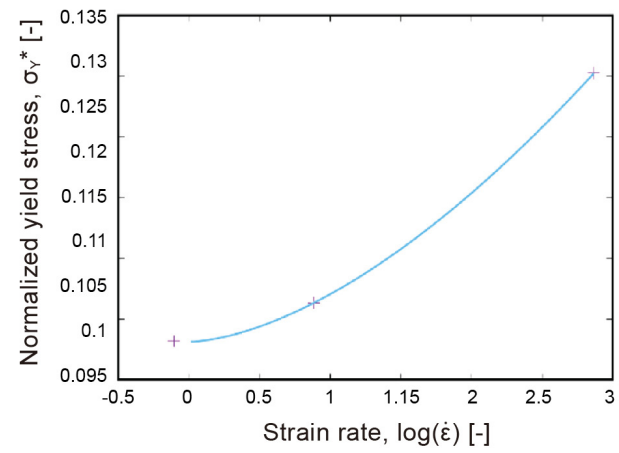

(b)

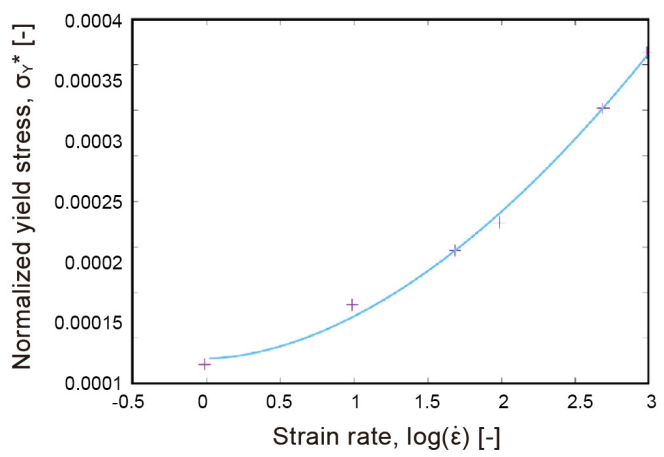

(d)

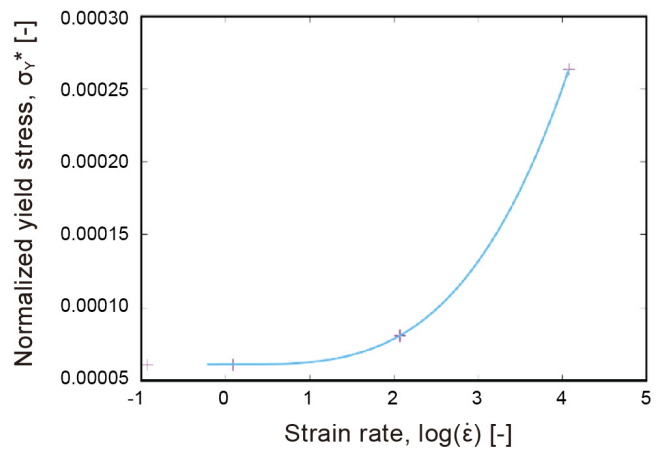

Fig. 11. Specimen size and strain rate effect on the normalized yield stress: (a) $\mathrm{L}_{c}=2 \mathrm{~nm}$, (b) $\mathrm{L}_{c}=10 \mathrm{~nm}$, (c) $\mathrm{L}_{c}=2 \mathrm{~nm}$, and (d) $\mathrm{L}_{c}=1.25 \mathrm{~nm}$.

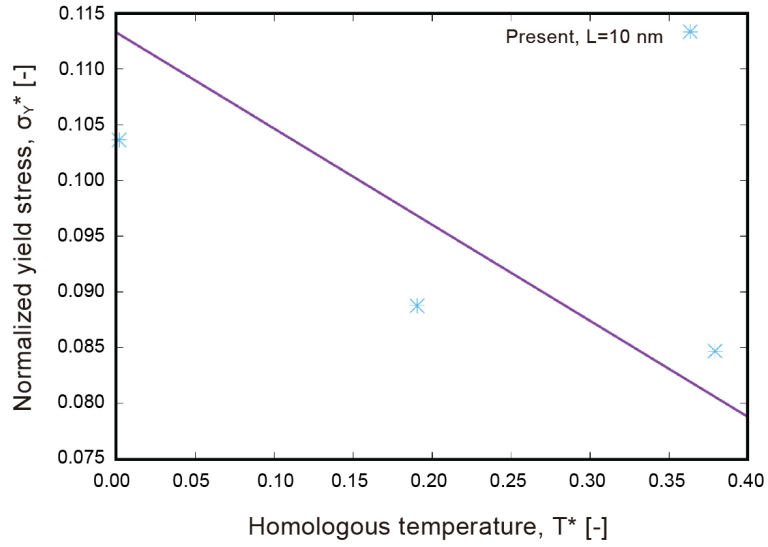

Fig. 12. Thermal effect on the normalized yield stress

at large specimen sizes. The yield stress with respect to strain rate was divided into two regions: a plateau stress region and an exponentially-increasing one. At high strain rates, the crystal structure including amorphous regions had a smoother zigzag curve after the yield point. It was found that the higher the temperature became, the lower the yield stress became, and the elastic modulus was lower with active atomic mobility. Next, material characterization was conducted based on these results. The thermal softening effect was expressed in terms of the elastic modulus. The other effects were expressed in terms of the normalized yield stress. These computational results can lead to the development of an in silico platform to characterize material properties, and MD simulation can lay the groundwork for multi-scale modeling and simulation.

\section{ACKNOWLEDGEMENTS}

This work was supported in part by the Defense Research Laboratory Program of the Defense Acquisition Program Administration and the Agency for Defense Development of Republic of Korea under the contract UD110089GD and the National Research Foundation of Korea (NRF) grant funded by the Korea government (MSIP) (No. 2011-0030075).

\section{REFERENCES}

1. J. L. Beeby, P. K. Bhattacharya, P. C. Gravelle, F. Koch, and D. J. Lockwood, Condensed Systems of Low Dimensionality, Plenum Press, New York (1991). 
2. S. Singh and S.-H. Park, Korean J. Met. Mater. 54, 347 (2016).

3. J. -B. Seol, Y.-T. Kim, B.-H. Kim, and C.-G. Park, Met. Mater. Int. 22, 34 (2016).

4. M. F. Horstemeyer, M. I. Baskes, and S. J. Plimpton, Acta Mater. 49, 4363 (2001).

5. D. Wang, J. Zhao, S. Hu, X. Yin, S. Liang, Y. Liu, and S. Deng, Nano Lett. 7, 1208 (2007).

6. W. Liang and M. Zhou, Nanotechnology 2, 452 (2003).

7. H. A. Wu, Eur. J. Mech. A-Solid 25, 370 (2006).

8. G. Rubio, N. Agrait, and S. Vieira, Phys. Rev. Lett. 76, 2302 (1996).

9. S. Dubois, L. Piraux, J. M. Gerge, K. Ounadjela, J. L. Duvail, and A. Fert, Phys. Rev. B 60, 477 (1999).

10. M. F. Horstemeyer and D. J. Bammann, Int. J. Plast. 26, 1310 (2010).

11. R. E. Rudd and J. Q. Broughton, Phys. Status Solidi B 217, 251 (2000).

12. E. Silva, C. Forst, J. Li, X. Lin, T. Zhu, and S. Yip, ESAIM-Math. Model. Numer. Model. Math. Anal. 41, 427 (2007).
13. F. F. Abraham, J. Q. Broughton, N. Bernstein, and E. Kaxiras, Comput. Phys. 12, 538 (1998).

14. S. Plimpton, J. Comput. Phys. 117, 1 (1995).

15. LAMMPS molecular dynamics simulator code homepage, http://lammps.sandia.gov.

16. M. S. Daw and M. I. Baskes, Phys. Rev. Lett. 50, 1285 (1983).

17. M. S. Daw and M. I. Baskes, Phys. Rev. B 29, 6443 (1984).

18. Y. Mishin, M. J. Mehl, D. A. Papaconstantopoulos, A. F. Voter, and J. D. Kress, Phys. Rev. B 63, 224106 (2001).

19. D. H. Tsai, J. Chem. Phys. 70, 1375 (1979).

20. A. Stukowski, Model. Simul. Mater. Sci. Eng. 18, 015012 (2010).

21. R. W. Hertzberg, Deformation and Fracture Mechanics of Engineering Materials, 4th ed., pp. 15, John Wiley \& Sons, New York (1996).

22. W. J. Kang, S. S. Cho, H. Huh, and D. T. Chung, Int. J. Veh. Des. 21, 424 (1999).

23. J. W. Edington, Philos. Mag. 19, 1189 (1969).

24. S. S. Brenner, J. Appl. Phys. 27, 1484 (1956). 